\title{
Selberg Integral Involving the Product of Multivariable Special Functions
}

\author{
By FY. AY. Ant
}

Abstract- The Selberg integral was an integral first evaluated by Selberg in 1944. The aim of the present paper is to estimate generalized Selberg integral. It involves the product of the general class of multivariable polynomials, multivariable I-function and modified multivariable $\mathrm{H}$-function. The result is believed to be new and is capable of giving a large number of integrals involving a variety of functions and polynomials as its cases. We shall see several corollaries and particular cases at the end.

Keywords: modified multivariable H-function, selberg integral, multivariable l-function, class of multivariable polynomials, $h$-function.

GJSFR-F Classification: FOR Code: MSC 2010: 33C60, 82C31

Strictly as per the compliance and regulations of:

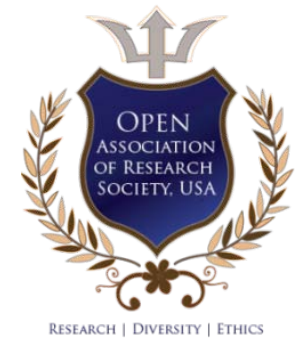

(c) 2018. FY. AY. Ant. This is a research/review paper, distributed under the terms of the Creative Commons AttributionNoncommercial 3.0 Unported License http://creativecommons.org/licenses/by-nc/3.0/), permitting all non commercial use, distribution, and reproduction in any medium, provided the original work is properly cited. 

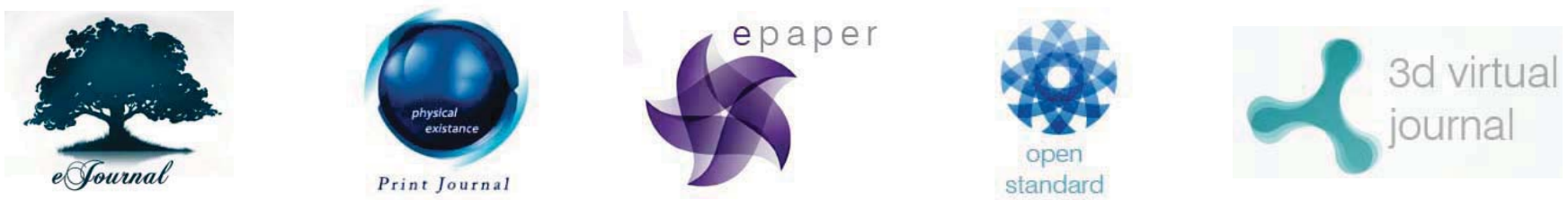

Abstract- The Selberg integral was an integral first evaluated by Selberg in 1944. The aim of the present paper is to estimate generalized Selberg integral. It involves the product of the general class of multivariable polynomials, multivariable l-function and modified multivariable $\mathrm{H}$-function. The result is believed to be new and is capable of giving a large number of integrals involving a variety of functions and polynomials as its cases. We shall see several corollaries and particular cases at the end.

Keywords: modified multivariable H-function, selberg integral, multivariable l-function, class of multivariable polynomials, h-function.

\section{Introduction and Prerequisites.}

The Selberg integral is the following integral first evaluated by Selberg [6] in 1944 :

$$
\begin{aligned}
S_{n}(a, b, c) & =\int_{0}^{1} \cdots \int_{0}^{1} \prod_{i=1}^{n} x_{i}^{a-1}\left(1-x_{i}\right)^{b-1} \prod_{1 \leqslant j<k \leqslant n}\left|x_{j}-x_{k}\right|^{2 c} \mathrm{~d} x_{1} \cdots \mathrm{d} x_{n} \\
& =\prod_{j=0}^{n-1} \frac{\Gamma(a+j c) \Gamma(b+j c) \Gamma(1+(j+1) c)}{\Gamma(a+b+(n-1+j) c) \Gamma(1+c)}
\end{aligned}
$$

where $\mathrm{n}$ is a positive integer, $a, b$ and $c$ are the complex number such that

$$
\operatorname{Re}(a)>0, \operatorname{Re}(b)>0, \operatorname{Re}(c)>\operatorname{Max}\left\{-\frac{1}{n},-\frac{\operatorname{Re}(a)}{n-1},-\frac{\operatorname{Re}(b)}{n-1}\right\}
$$

We refer the reader to Forrester and Warnaar's exposition [2] for the history and importance of the Selberg integral.

In this document, we evaluate a generalized Selberg integral involving the product of the multivariable I-function defined by Prasad [4], modified multivariable Hfunction defined by Prasad and Singh [5] and class of multivariable polynomials defined by Srivastava [7].

The generalized multivariable polynomials defined by Srivastava [7], is given in the following manner:

$$
S_{N_{1}, \cdots, N_{v}}^{\mathfrak{M}_{1}, \cdots, \mathfrak{M}_{\mathfrak{v}}}\left[y_{1}, \cdots, y_{v}\right]=\sum_{K_{1}=0}^{\left[N_{1} / \mathfrak{M}_{1}\right]} \cdots \sum_{K_{v}=0}^{\left[N_{v} / \mathfrak{M}_{\mathfrak{v}}\right]} \frac{\left(-N_{1}\right)_{\mathfrak{M}_{1} K_{1}}}{K_{1} !} \cdots \frac{\left(-N_{v}\right)_{\mathfrak{M}_{\mathfrak{v}} K_{v}}}{K_{v} !} A\left[N_{1}, K_{1} ; \cdots ; N_{v}, K_{v}\right] y_{1}^{K_{1}} \cdots y_{v}^{K_{v}}
$$

where $\mathfrak{M}_{1}, \cdots, \mathfrak{M}_{\mathfrak{v}}$ are arbitrary positive integers and the coefficients $A\left[N_{1}, K_{1} ; \cdots ; N_{v}, K_{v}\right]$ are constants Real or complex. On suitably specializing the quantities, $A\left[N_{1}, K_{1} ; \cdots ; N_{v}, K_{v}\right], S_{N_{1}, \cdots, N_{v}}^{\mathfrak{M}_{1}, \cdots, \mathfrak{M}_{\mathfrak{b}}}\left[y_{1}, \cdots, y_{v}\right]$ yields a Number of known polynomials, the 
Laguerre polynomials, the Jacobi polynomials, and several other ([10], page. 158-161]. We shall note.

$$
a_{v}=\frac{\left(-N_{1}\right)_{\mathfrak{M}_{1} K_{1}}}{K_{1} !} \cdots \frac{\left(-N_{v}\right)_{\mathfrak{M}_{\mathfrak{v}} K_{v}}}{K_{v} !} A\left[N_{1}, K_{1} ; \cdots ; N_{v}, K_{v}\right]
$$

The multivariable I-function of r-variables is defined in term of multiple MellinBarnes types integral:

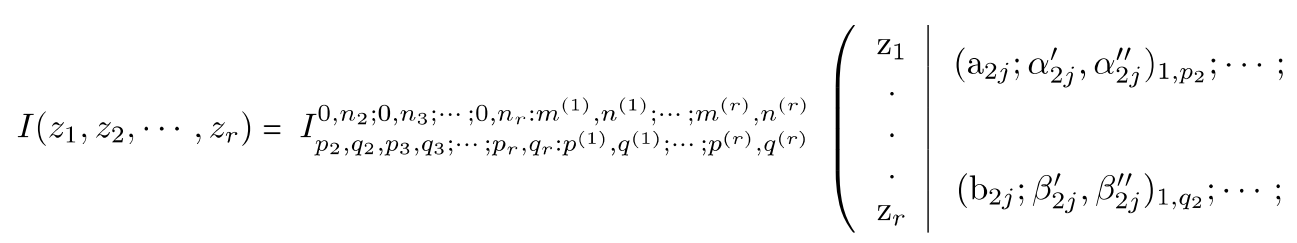

$$
\begin{array}{r}
\left(\mathrm{a}_{r j} ; \alpha_{r j}^{(1)}, \cdots, \alpha_{r j}^{(r)}\right)_{1, p_{r}}:\left(a_{j}^{(1)}, \alpha_{j}^{(1)}\right)_{1, p^{(1)}} ; \cdots ;\left(a_{j}^{(r)}, \alpha_{j}^{(r)}\right)_{1, p^{(r)}} \\
\left.\left(\mathrm{b}_{r j} ; \beta_{r j}^{(1)}, \cdots, \beta_{r j}^{(r)}\right)_{1, q_{r}}:\left(b_{j}^{(1)}, \beta_{j}^{(1)}\right)_{1, q^{(1)}} ; \cdots ;\left(b_{j}^{(r)}, \beta_{j}^{(r)}\right)_{1, q^{(r)}}\right) \\
=\frac{1}{(2 \pi \omega)^{r}} \int_{L_{1}} \cdots \int_{L_{r}} \phi\left(s_{1}, \cdots, s_{r}\right) \prod_{i=1}^{r} \phi_{i}\left(s_{i}\right) z_{i}^{s_{i}} \mathrm{~d} s_{1} \cdots \mathrm{d} s_{r}
\end{array}
$$

where

$$
\phi_{i}\left(s_{i}\right)=\frac{\prod_{j=1}^{m^{(i)}} \Gamma\left(b_{j}^{(i)}-\beta_{j}^{(i)} s_{i}\right) \prod_{j=1}^{n^{(i)}} \Gamma\left(1-a_{j}^{(i)}+\alpha_{j}^{(i)} s_{i}\right)}{\prod_{j=m^{(i)}+1}^{q^{(i)}} \Gamma\left(1-b_{j}^{(i)}+\beta_{j}^{(i)} s_{i}\right) \prod_{j=n^{(i)}+1}^{p^{(i)}} \Gamma\left(a_{j}^{(i)}-\alpha_{j}^{(i)} s_{i}\right)}, i=1, \cdots, r
$$

and

$$
\begin{aligned}
& \phi\left(s_{1}, \cdots, s_{r}\right)= \frac{\prod_{j=1}^{n_{2}} \Gamma\left(1-a_{2 j}+\sum_{i=1}^{2} \alpha_{2 j}^{(i)} s_{i}\right) \prod_{j=1}^{n_{3}} \Gamma\left(1-a_{3 j}+\sum_{i=1}^{3} \alpha_{3 j}^{(i)} s_{i}\right) \cdots}{\prod_{j=n_{2}+1}^{p_{2}} \Gamma\left(a_{2 j}-\sum_{i=1}^{2} \alpha_{2 j}^{(i)} s_{i}\right) \prod_{j=n_{3}+1}^{p_{3}} \Gamma\left(a_{3 j}-\sum_{i=1}^{3} \alpha_{3 j}^{(i)} s_{i}\right) \cdots} \\
& \frac{\cdots \prod_{j=1}^{n_{r}} \Gamma\left(1-a_{r j}+\sum_{i=1}^{r} \alpha_{r j}^{(i)} s_{i}\right)}{\cdots \prod_{j=n_{r}+1}^{p_{r}} \Gamma\left(a_{r j}-\sum_{i=1}^{r} \alpha_{r j}^{(i)} s_{i}\right) \prod_{j=1}^{q_{2}} \Gamma\left(1-b_{2 j}-\sum_{i=1}^{2} \beta_{2 j}^{(i)} s_{i}\right)} \\
& \times \frac{1}{\prod_{j=1}^{q_{3}} \Gamma\left(1-b_{3 j}+\sum_{i=1}^{3} \beta_{3 j}^{(i)} s_{i}\right) \cdots \prod_{j=1}^{q_{r}} \Gamma\left(1-b_{r j}-\sum_{i=1}^{r} \beta_{r j}^{(i)} s_{i}\right)}
\end{aligned}
$$

About the above integrals and these existence and convergence conditions, see Prasad [4] for more details. Throughout the present document, we assume that the existence and convergence conditions of the multivariable I-function. We have:

$\left|\arg z_{i}\right|<\frac{1}{2} \Omega_{i} \pi$, where

$$
\Omega_{i}=\sum_{k=1}^{n^{(i)}} \alpha_{k}^{(i)}-\sum_{k=n^{(i)}+1}^{p^{(i)}} \alpha_{k}^{(i)}+\sum_{k=1}^{m^{(i)}} \beta_{k}^{(i)}-\sum_{k=m^{(i)}+1}^{q^{(i)}} \beta_{k}^{(i)}+\left(\sum_{k=1}^{n_{2}} \alpha_{2 k}^{(i)}-\sum_{k=n_{2}+1}^{p_{2}} \alpha_{2 k}^{(i)}\right)+\cdots+
$$




$$
\left(\sum_{k=1}^{n_{r}} \alpha_{r k}^{(i)}-\sum_{k=n_{r}+1}^{p_{r}} \alpha_{r k}^{(i)}\right)-\left(\sum_{k=1}^{q_{2}} \beta_{2 k}^{(i)}+\sum_{k=1}^{q_{3}} \beta_{3 k}^{(i)}+\cdots+\sum_{k=1}^{q_{r}} \beta_{r k}^{(i)}\right)
$$

where $i=1, \cdots, r$

The complex numbers $z_{i}$ are not zero. Throughout this document, we assume the existence and absolute convergence conditions of the multivariable I-function. We may establish the asymptotic expansion in the following convenient form:

$$
\begin{aligned}
& I\left(z_{1}, \cdots, z_{r}\right)=0\left(\left|z_{1}\right|^{\alpha_{1}}, \cdots,\left|z_{r}\right|^{\alpha_{r}}\right), \max \left(\left|z_{1}\right|, \cdots,\left|z_{r}\right|\right) \rightarrow 0 \\
& I\left(z_{1}, \cdots, z_{r}\right)=0\left(\left|z_{1}\right|^{\beta_{1}}, \cdots,\left|z_{r}\right|^{\beta_{r}}\right), \min \left(\left|z_{1}\right|, \cdots,\left|z_{r}\right|\right) \rightarrow \infty
\end{aligned}
$$

where $k=1, \cdots, r: \alpha_{k}^{\prime}=\min \left[\operatorname{Re}\left(b_{j}^{(k)} / \beta_{j}^{(k)}\right)\right], j=1, \cdots, m^{(k)}$ and

$$
\beta_{k}^{\prime}=\max \left[\operatorname{Re}\left(\left(a_{j}^{(k)}-1\right) / \alpha_{j}^{(k)}\right)\right], j=1, \cdots, n^{(k)}
$$

If all the poles of (1.7) are simples, then the integral (1.6) can be evaluated with the help of the residue theorem to give

$$
I\left(z_{1}, \cdots, z_{r}\right)=\sum_{G_{i}=1}^{m^{(i)}} \sum_{g_{i}=0}^{\infty} \phi \frac{\prod_{i=1}^{r} \phi_{i} z_{i}^{\eta_{G_{i}, g_{i}}}(-)^{\sum_{i=1}^{r} g_{i}}}{\prod_{i=1}^{r} \delta_{G^{(i)}}^{(i)} \prod_{i=1}^{r} g_{i} !}
$$

where

$$
\begin{aligned}
\phi & =\phi\left(\eta_{G_{1}, g_{1}}, \cdots, \eta_{G_{r}, g_{r}}\right), \phi_{i}=\phi_{i}\left(\eta_{G_{i}, g_{i}}\right), i=1, \cdots, r \\
\eta_{G_{i}, g_{i}} & =\frac{d_{g_{i}}^{(i)}+G_{i}}{\delta_{g_{i}}^{(i)}} \text { for } i=1, \cdots, r \text { and } \sum_{G_{i}=1}^{m^{(i)}} \sum_{g_{i}=0}^{\infty}=\sum_{G_{1}, \cdots, G_{r}=1}^{m^{(1)}, \cdots, m^{(r)}} \sum_{g_{1}, \cdots, g_{r}=0}^{\infty}
\end{aligned}
$$

which is valid under the following conditions: $\epsilon_{M_{i}}^{(i)}\left[p_{j}^{(i)}+p_{i}^{\prime}\right] \neq \epsilon_{j}^{(i)}\left[p_{M_{i}}+g_{i}\right] . \phi_{i}$ and $\phi$ are are given by (1.5) and (1.6) respectively.

The modified H-function studied by Prasad and Singh [5] generalizes the multivariable H-function defined by Srivastava and Panda [8,9]. It is defined in term of multiple Mellin-Barnes types integral:

$$
\begin{aligned}
& H\left(z_{1}^{\prime}, \cdots, z_{s}^{\prime}\right)=H_{\mathbf{p}, \mathbf{q}: \mid R: p_{1}, q_{1} ; \cdots, p_{s}, q_{s}}^{\mathbf{m}, \mathbf{R}: \mid m_{1}, n_{1} ; \cdots, m_{s}}\left(\begin{array}{c|c}
\mathrm{z}_{1}^{\prime} & \left(\mathrm{a}_{j} ; \alpha_{j}^{(1)}, \cdots, \alpha_{j}^{(s)}\right)_{1, \mathbf{p}}: \\
\cdot & \cdot \\
\cdot & \cdot \\
\cdot & \left(\mathrm{b}_{j} ; \beta_{j}^{(1)}, \cdots, \beta_{j}^{(s)}\right)_{1, \mathbf{q}}:
\end{array}\right. \\
& \begin{array}{c}
\left(\mathrm{e}_{j} ; u_{j}^{(1)} g_{j}^{(1)}, \cdots, u_{j}^{(s)} g_{j}^{(s)}\right)_{1, \mathbf{R}}:\left(c_{j}^{(1)} ; \gamma_{j}^{(1)}\right)_{1, p_{1}}, \cdots,\left(c_{j}^{(s)} ; \gamma_{j}^{(s)}\right)_{1, p_{s}} \\
\cdot \\
\left.\cdot \cdot{ }^{(1)} ; \delta_{j}^{(1)}\right)_{1, q_{1}}, \cdots,\left(d_{j}^{(s)} ; \delta_{j}^{(s)}\right)_{1, q_{s}} \\
\left(\mathrm{l}_{j} ; U_{j}^{(1)} f_{j}^{(1)}, \cdots, U_{j}^{(s)} f_{j}^{(r)}\right)_{1, R}:\left(d_{j}\right)
\end{array} \\
& =\frac{1}{(2 \pi \omega)^{s}} \int_{L_{1}^{\prime}} \cdots \int_{L_{s}^{\prime}} \theta\left(t_{1}, \cdots, t_{s}\right) \prod_{i=1}^{s} \theta_{i}\left(t_{i}\right) z_{i}^{\prime t_{i}} \mathrm{~d} t_{1} \cdots \mathrm{d} t_{s}
\end{aligned}
$$


where $\theta\left(t_{1}, \cdots, t_{s}\right), \theta_{i}\left(t_{i}\right), i=1, \cdots, s$ are given by:

$$
\begin{gathered}
\theta\left(t_{1}, \cdots, t_{s}\right)=\frac{\prod_{j=1}^{\mathbf{m}} \Gamma\left(b_{j}-\sum_{i=1}^{s} \beta_{j}^{(i)} t_{i}\right) \prod_{j=1}^{\mathbf{n}} \Gamma\left(1-a_{j}+\sum_{i=1}^{s} \alpha_{j}^{(i)} t_{j}\right)}{\prod_{j=\mathbf{n}+1}^{\mathbf{p}} \Gamma\left(a_{j}-\sum_{i=1}^{s} \alpha_{j}^{(i)} t_{j}\right) \prod_{j=\mathbf{m}+1}^{\mathbf{q}} \Gamma\left(1-b_{j}+\sum_{i=1}^{s} \beta_{j}^{(i)} t_{j}\right)} \\
\frac{\prod_{j=1}^{\mathbf{R}} \Gamma\left(e_{j}+\sum_{i=1}^{s} u_{j}^{(i)} g_{j}^{(i)} t_{i}\right)}{\prod_{j=1}^{R} \Gamma\left(l_{j}+\sum_{i=1}^{s} U_{j}^{(i)} f_{j}^{(i)} t_{i}\right)} \\
\theta_{i}\left(t_{i}\right)=\frac{\prod_{j=1}^{n_{i}} \Gamma\left(1-c_{j}^{(i)}+\gamma_{j}^{(i)} t_{i}\right) \prod_{j=1}^{m_{i}} \Gamma\left(d_{j}^{(i)}-\delta_{j}^{(i)} t_{i}\right)}{\prod_{j=n_{i}+1}^{p_{i}} \Gamma\left(c_{j}^{(i)}-\gamma_{j}^{(i)} t_{i}\right) \prod_{j=m_{i}+1}^{q_{i}} \Gamma\left(1-d_{j}^{(i)}+\delta_{j}^{(i)} t_{i}\right)}
\end{gathered}
$$

The integrals (1.14) converges absolutely if

$$
\left|\arg z_{i}^{\prime}\right|<\frac{1}{2} U_{i} \pi \quad(i=1, \cdots, s)
$$

with

$$
\begin{aligned}
U_{i}=\sum_{j=1}^{\mathbf{m}} \beta_{j}^{(i)}- & \sum_{j=\mathbf{m}+1}^{\mathbf{q}} \beta_{j}^{(i)}+\sum_{j=1}^{\mathbf{n}} \alpha_{j}^{(i)}-\sum_{j=\mathbf{n}+1}^{\mathbf{p}} \alpha_{j}^{(i)}+\sum_{j=1}^{m_{i}} \delta_{j}^{(i)}-\sum_{j=1+m_{i}}^{q_{i}} \delta_{j}^{(i)} \\
& +\sum_{j=1}^{n_{i}} \gamma_{j}^{(i)}-\sum_{j=n_{i}+1}^{p_{i}} \gamma_{j}^{(i)}+\sum_{j=1}^{\mathbf{R}} g_{j}^{(i)}-\sum_{j=1}^{R} f_{j}^{(i)}>0(i=1, \cdots, s)
\end{aligned}
$$

For more details, see Prasad and Singh [5].

\section{REQUIRED INTEGRAL}

We have the following integrals, see Andrew and R. Askey for more details ([1], p. 402). Lemma.

$\int_{0}^{1} \cdots \quad \prod_{i=1}^{k} x_{i} \prod_{i=1}^{n} x_{i}^{a-1}\left(1-x_{i}\right)^{b-1} \prod_{1 \leqslant j<k \leqslant n}\left|x_{j}-x_{k}\right|^{2 c} \mathrm{~d} x_{1} \cdots \mathrm{d} x_{n}=\prod_{i=1}^{k} \frac{(a+(n-i) c)}{(a+b+(2 n-i-1) c)} S_{n}(a, b, c)$

Where $\operatorname{Re}(a)>0, \operatorname{Re}(b)>0, \operatorname{Re}(c)>\operatorname{Max}\left\{-\frac{1}{n},-\frac{\operatorname{Re}(a)}{n-1},-\frac{\operatorname{Re}(b)}{n-1}\right\}$ and $k \leqslant n . S_{n}(a, b, c)$ is defined by (1.1).

\section{MAIN INTEGRAL}

Let

$$
\begin{aligned}
X_{u, v, w}\left(x_{1}, \cdots, x_{n}\right) & =\prod_{i=1}^{n} x_{i}^{u}\left(1-x_{i}\right)^{v} \prod_{1 \leqslant j<k \leqslant n}\left|x_{j}-x_{k}\right|^{2 w} \\
X & =m_{1}, n_{1} ; \cdots ; m_{s}, n_{s} \\
Y & =p_{1}, q_{1} ; \cdots ; p_{s}, q_{s}
\end{aligned}
$$




$$
\begin{aligned}
& \mathbb{A}=\left(a_{j} ; \alpha_{j}^{(1)}, \cdots, \alpha_{j}^{(s)}\right)_{1, \mathbf{p}} ;\left(\mathrm{e}_{j} ; u_{j}^{(1)} g_{j}^{(1)}, \cdots, u_{j}^{(s)} g_{j}^{(s)}\right)_{1, \mathbf{R}}: \mathrm{C}=\left(\mathrm{c}_{j}^{(1)} ; \gamma_{j}^{(1)}\right)_{1, p_{1}} ; \cdots,\left(c_{j}^{(s)} ; \gamma_{j}^{(s)}\right)_{1, p_{s}} \\
& \mathbb{B}=\left(b_{j} ; \beta_{j}^{(1)}, \cdots, \beta_{j}^{(s)}\right)_{1, \mathbf{q}} ; \quad\left(\mathrm{l}_{j} ; U_{j}^{(1)} f_{j}^{(1)}, \cdots, U_{j}^{(s)} f_{j}^{(s)}\right)_{1, R}: D=\left(d_{j}^{(1)} ; \delta_{j}^{(1)}\right)_{1, q_{1}} ; \cdots ;\left(d_{j}^{(s)} ; \delta_{j}^{(s)}\right)_{1, q_{s}}
\end{aligned}
$$

In this section, we establish the general Selberg integral about the product of a class of multivariable polynomials, multivariable I-function and modified H-function of several variables.

\section{Theorem.}

$$
\begin{aligned}
& \int_{0}^{1} \cdots \int_{0}^{1} \prod_{i=1}^{k} x_{i} \prod_{i=1}^{n} x_{i}^{a-1}\left(1-x_{i}\right)^{b-1} \prod_{1 \leqslant j<k \leqslant n}\left|x_{j}-x_{k}\right|^{2 c} S_{N_{1}, \cdots, N_{v}}^{\mathfrak{M}_{1}, \cdots, \mathfrak{M}_{\mathfrak{v}}}\left(\begin{array}{c}
\mathrm{y}_{1} X_{\alpha_{1}, \beta_{1}, \gamma_{1}}\left(x_{1}, \cdots, x_{n}\right) \\
\dot{ } \\
\mathrm{y}_{v} X_{\alpha_{v}, \beta_{v}, \gamma_{v}}\left(x_{1}, \cdots, x_{n}\right)
\end{array}\right)
\end{aligned}
$$

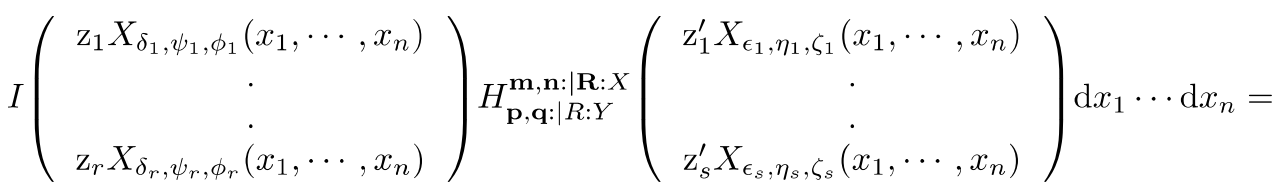

$$
\begin{aligned}
& \sum_{K_{1}=0}^{\left[N_{1} / \mathfrak{M}_{\mathbf{1}}\right]} \cdots \sum_{K_{v}=0}^{\left[N_{v} / \mathfrak{M}_{\mathfrak{v}}\right]} \sum_{G_{i}=1}^{m^{(i)}} \sum_{g_{i}=0}^{\infty} \phi \frac{\prod_{i=1}^{r} \phi_{i} z_{i}^{\eta_{G_{i}}, g_{i}}(-)^{\sum_{i=1}^{r} g_{i}}}{\prod_{i=1}^{r} \delta_{G^{(i)}}^{(i)} \prod_{i=1}^{r} g_{i} !} a_{v} y_{1}^{R_{1}} \cdots y_{v}^{R_{v}} \\
& H_{\mathbf{p}+3 n+2 k, \mathbf{q}+2 n+2 k: \mid R: Y}^{\mathbf{m}, \mathbf{n}+3 n+2 k: \mathbf{R}: X}\left(\begin{array}{c|c}
\mathrm{z}_{1}^{\prime} & \mathrm{A}_{1}, A_{2}, A_{3}, A_{4}, A_{5}, \mathbb{A}: C \\
\cdot & \cdot \\
\cdot & \cdot \\
\cdot & \mathbb{B}, \mathrm{B}_{1}, B_{2}, B_{3}, B_{4}: D
\end{array}\right)
\end{aligned}
$$

where

$$
\begin{aligned}
& A_{1}=\left[1-a-\sum_{i=1}^{v} K_{i} \alpha_{i}-\sum_{i=1}^{r} \eta_{G_{i}, g_{i}} \delta_{i}-j\left(c+\sum_{i=1}^{v} \gamma_{i} K_{i}+\sum_{i=1}^{r} \phi_{i} \eta_{G_{i}, g_{i}}\right) ; \epsilon_{1}+j \zeta_{1}, \cdots, \epsilon_{s}+j \zeta_{s}\right]_{0, n-1} \\
& A_{2}=\left[1-b-\sum_{i=1}^{v} K_{i} \beta_{i}-\sum_{i=1}^{r} \eta_{G_{i}, g_{i}} \psi_{i}-j\left(c+\sum_{i=1}^{v} \gamma_{i} K_{i}+\sum_{i=1}^{r} \phi_{i} \eta_{G_{i}, g_{i}}\right) ; \eta_{1}+j \zeta_{1}, \cdots, \eta_{s}+j \zeta_{s}\right]_{0, n-1} \\
& \left.A_{3}=\left[-j\left(c+\sum_{i=1}^{v} \gamma_{i} K_{i}+\sum_{i=1}^{r} \phi_{i} \eta_{G_{i}, g_{i}}\right) ; j \zeta_{1}, \cdots, j \zeta_{s}\right)\right]_{1, n} \\
& A_{4}=\left[-a-\sum_{i=1}^{v} K_{i} \alpha_{i}-\sum_{i=1}^{r} \eta_{G_{i}, g_{i}} \delta_{i}-(n-j)\left(c+\sum_{i=1}^{v} \gamma_{i} K_{i}+\sum_{i=1}^{r} \phi_{i} \eta_{G_{i}, g_{i}}\right) ;\right. \\
& \left.\epsilon_{1}+(n-j) \zeta_{1}, \cdots, \epsilon_{s}+(n-j) \zeta_{s}\right]_{1, k} \\
& A_{5}=\left[1-a-b-\sum_{i=1}^{v} K_{i}\left(\alpha_{i}+\beta_{i}\right)-\sum_{j=1}^{r} \eta_{G_{j}, g_{j}}\left(\delta_{j}+\psi_{j}\right)-(2 n-j-1)\left(c+\sum_{i=1}^{v} \gamma_{i} K_{i}+\sum_{i=1}^{r} \phi_{i} \eta_{G_{i}, g_{i}}\right) ;\right.
\end{aligned}
$$


$\left.\epsilon_{1}+\eta_{1}+(2 n-j-1) \zeta_{1}, \cdots \epsilon_{s}+\eta_{s}+(2 n-j-1) \zeta_{s}\right]_{1, k}$

$B_{1}=\left(-c-\sum_{i=1}^{v} K_{i} \gamma_{i}-\sum_{i=1}^{r} \phi_{i} \eta_{G_{i}, g_{i}} ; \zeta_{1}, \cdots, \zeta_{s}\right), \cdots,\left(-c-\sum_{i=1}^{v} \gamma_{i} R_{i}-\sum_{i=1}^{r} \phi_{i} \eta_{G_{i}, g_{i}} ; \zeta_{1}, \cdots, \zeta_{s}\right)$

$B_{2}=\left[1-a-\sum_{i=1}^{v} K_{i} \alpha_{i}-\sum_{i=1}^{r} \eta_{G_{i}, g_{i}} \delta_{i}-(n-j)\left(c+\sum_{i=1}^{v} \gamma_{i} K_{i}+\sum_{i=1}^{r} \phi_{i} \eta_{G_{i}, g_{i}}\right) ;\right.$

$\left.\epsilon_{1}+(n-j) \zeta_{1}, \cdots, \epsilon_{s}+(n-j) \zeta_{s}\right]_{1, k}$

$B_{3}=\left[-a-b-\sum_{i=1}^{v} K_{i}\left(\alpha_{i}+\beta_{i}\right)-\sum_{i=1}^{r} \eta_{G_{i}, g_{i}}\left(\delta_{i}+\psi_{i}\right)-(2 n-j-1)\left(c+\sum_{i=1}^{v} \gamma_{i} K_{i}+\sum_{i=1}^{r} \phi_{i} \eta_{G_{i}, g_{i}}\right)\right.$

$\left.\epsilon_{1}+\eta_{1}+(2 n-j-1) \zeta_{1}, \cdots \epsilon_{s}+\eta_{s}+(2 n-j-1) \zeta_{s}\right]_{1, k}$

$B_{4}=\left[1-a-b-\sum_{i=1}^{v} K_{i}\left(\alpha_{i}+\beta_{i}\right)-\sum_{i=1}^{r} \eta_{G_{i}, g_{i}}\left(\delta_{i}+\psi_{j}\right)-(n+j-1)\left(c+\sum_{i=1}^{v} \gamma_{i} K_{i}+\sum_{i=1}^{r} \phi_{i} \eta_{G_{i}, g_{i}}\right)\right.$

$\left.\epsilon_{1}+\eta_{1}+(n+j-1) \zeta_{1}, \cdots \epsilon_{s}+\eta_{s}+(n+j-1) \zeta_{s}\right]_{0, n-1}$

Provided

$\min \left\{\alpha_{i}, \beta_{i}, \gamma_{i}, \delta_{j}, \psi_{j}, \phi_{j}, \epsilon_{l}, \eta_{l}, \zeta_{l}\right\}>0, i=1, \cdots, v, j=1, \cdots, r, l=1, \cdots, s ; a, b, c \in \mathbb{C}$

$$
\begin{aligned}
& A=\operatorname{Re}\left(a+\sum_{i=1}^{r} \delta_{i} \eta_{G_{i}, g_{i}}\right)+\sum_{i=1}^{s} \epsilon_{i} \min _{1 \leqslant j \leqslant m_{i}} \operatorname{Re}\left(\frac{d_{j}^{(i)}}{\delta_{j}^{(i)}}\right)>0 \\
& B=\operatorname{Re}\left(b+\sum_{i=1}^{r} \psi_{i} \eta_{G_{i}, g_{i}}\right)+\sum_{i=1}^{s} \eta_{i} \min _{1 \leqslant j \leqslant m_{i}} \operatorname{Re}\left(\frac{d_{j}^{(i)}}{\delta_{j}^{(i)}}\right)>0 \\
& C=\operatorname{Re}\left(c+\sum_{i=1}^{r} \phi_{i} \eta_{G_{i}, g_{i}}\right)+\sum_{i=1}^{s} \zeta_{i} \min _{1 \leqslant j \leqslant m_{i}} \operatorname{Re}\left(\frac{d_{j}^{(i)}}{\delta_{j}^{(i)}}\right)>\operatorname{Max}\left\{-\frac{1}{n},-\frac{A}{n-1},-\frac{B}{n-1}\right\} \text { and } k \leqslant n .
\end{aligned}
$$

$\left|\arg \left(z_{i} X_{\delta_{i}, \psi_{i}, \phi_{i}}\left(x_{1}, \cdots, x_{n}\right)\right)\right|<\frac{1}{2} \Omega_{i} \pi$, where $\Omega_{i}$ is defined by $(1.7)$ for $i=1, \cdots, r$

$\left|\arg \left(z_{i}^{\prime} X_{\epsilon_{i}, \eta_{i}, \zeta_{i}}\left(x_{1}, \cdots, x_{n}\right)\right)\right|<\frac{1}{2} U_{i} \pi$, where $U_{i}$ is defined by $(1.15)$ for $i=1, \cdots, s$

the multiple series on the left-hand side of (3.6) converges absolutely.

\section{Proof}

To evaluate the integrals (3.6), first, we replace the class of multivariable polynomials $S_{N_{1}, \cdots, N_{v}}^{\mathfrak{M}_{1}, \cdots, \mathfrak{M}_{\mathfrak{v}}}[$ [.] and multivariable I-function occurring on the left-hand side of your integral in term of series with the help of (1.2) and (1.8) Respectively. Now we express the modified multivariable $\mathrm{H}$-function in Mellin-Barnes contour integrals by using (1.11). Nest, we change the order of the $\left(t_{1}, \cdots, t_{s}\right)$-integrals and $\left(x_{1}, \cdots, x_{n}\right)$-integrals, (which is justified under the conditions stated), we obtain the following result (say L.H.S.): 


$$
\begin{aligned}
& L . H . S=\sum_{K_{1}=0}^{\left[N_{1} / \mathfrak{M}_{1}\right]} \cdots \sum_{K_{v}=0}^{\left[N_{v} / \mathfrak{M}_{\mathfrak{v}}\right]} \sum_{G_{i}=1}^{m^{(i)}} \sum_{g_{i}=0}^{\infty} \phi \frac{\prod_{i=1}^{r} \phi_{i} z_{i}^{\eta_{G_{i}, g_{i}}}(-)^{\sum_{i=1}^{r} g_{i}}}{\prod_{i=1}^{r} \delta_{G^{(i)}}^{(i)} \prod_{i=1}^{r} g_{i} !} \\
& a_{v} y_{1}^{K_{1}} \cdots y_{v}^{K_{v}} \frac{1}{(2 \pi \omega)^{s}} \int_{L_{1}^{\prime}} \cdots \int_{L_{s}^{\prime}} \theta\left(t_{1}, \cdots, t_{s}\right) \prod_{l^{\prime}=1}^{s} \theta_{l^{\prime}}\left(t_{l^{\prime}}\right) z_{l^{\prime}}^{\prime t_{l^{\prime}}}\left[\int_{0}^{1} \cdots \int_{0}^{1} \prod_{i=1}^{k} x_{i}\right. \\
& \prod_{i=1}^{n} x_{i}^{a-1}+\sum_{l=1}^{v} K_{l} \alpha_{l}+\sum_{l=1}^{r} \eta_{G_{l}, g_{l}} \delta_{l}+\sum_{l=1}^{s} \epsilon_{l} t_{l}\left(1-x_{i}\right)^{b-1}+\sum_{l=1}^{v} K_{l} \beta_{l}+\sum_{l=1}^{r} \eta_{G_{l}, g_{l}} \psi_{l}+\sum_{l=1}^{s} \eta_{l} t_{l} \\
& \left.\prod_{1 \leqslant j<k \leqslant n}\left|x_{j}-x_{k}\right|^{2\left(c+\sum_{l=1}^{v} K_{l} \gamma_{l}+\sum_{l=1}^{r} \eta_{G_{l}, g_{l}} \phi_{l}+\sum_{l=1}^{s} \zeta_{l} t_{l}\right)} \mathrm{d} x_{1} \cdots \mathrm{d} x_{n}\right] \mathrm{d} t_{1} \cdots \mathrm{d} t_{s}
\end{aligned}
$$

Now we evaluate the inner $\left(x_{1}, \cdots, x_{n}\right)$-integrals with the help of the lemma and reinterpreting the Mellin-Barnes contour integrals thus obtained regarding modified $\mathrm{H}$ function of $s$-variables, we arrive at the required result after algebraic manipulations.

\section{Special Cases}

The multivariable polynomial vanishes and the multivariable I-function reduces to H-function defined by Fox [3], we obtain:

\section{Corollary 1.}

$$
\begin{gathered}
\int_{0}^{1} \cdots \int_{0}^{1} \prod_{i=1}^{k} x_{i} \prod_{i=1}^{n} x_{i}^{a-1}\left(1-x_{i}\right)^{b-1} \prod_{1 \leqslant j<k \leqslant n}\left|x_{j}-x_{k}\right|^{2 c} H\left(\mathrm{z}_{1} X_{\delta_{1}, \psi_{1}, \phi_{1}}\left(x_{1}, \cdots, x_{n}\right)\right) \\
H_{\mathbf{p}, \mathbf{q}: \mid R: Y}^{\mathbf{m}: \mathbf{R}: X}\left(\begin{array}{c}
\mathrm{z}_{1}^{\prime} X_{\epsilon_{1}, \eta_{1}, \zeta_{1}}\left(x_{1}, \cdots, x_{n}\right) \\
\cdot \\
\cdot \\
\mathrm{z}_{s}^{\prime} X_{\epsilon_{s}, \eta_{s}, \zeta_{s}}\left(x_{1}, \cdots, x_{n}\right)
\end{array}\right) \mathrm{d} x_{1} \cdots \mathrm{d} x_{n}=\sum_{G=1}^{m^{(1)}} \sum_{g=0}^{\infty} \phi_{1} \frac{z_{1}^{\eta_{G, g}}(-)^{g}}{\delta_{G} g !} \\
H_{\mathbf{p}+3 n+2 k, \mathbf{q}+2 n+2 k: \mid R: Y}^{\mathbf{m}, \mathbf{n}+3 n+2 k: \mid \mathbf{R}: X}\left(\begin{array}{c}
\mathrm{z}_{1}^{\prime} \\
\cdot \\
\cdot \\
\cdot \\
\mathrm{z}_{1}^{\prime}, A_{2}^{\prime}, A_{3}^{\prime}, A_{4}^{\prime}, A_{5}^{\prime}, \mathbb{A}: C \\
\cdot \\
\mathbb{B}, \mathrm{B}_{1}^{\prime}, B_{2}^{\prime}, B_{3}^{\prime}, B_{4}^{\prime}: D
\end{array}\right)
\end{gathered}
$$

where

$$
\begin{aligned}
& A_{1}^{\prime}=\left[1-a-\eta_{G, g} \delta_{1}-j\left(c+\phi_{1} \eta_{G, g}\right) ; \epsilon_{1}+j \zeta_{1}, \cdots, \epsilon_{s}+j \zeta_{s}\right]_{0, n-1} \\
& A_{2}^{\prime}=\left[1-b-\eta_{G, g} \psi_{1}-j\left(c+\phi_{1} \eta_{G, g}\right) ; \eta_{1}+j \zeta_{1}, \cdots, \eta_{s}+j \zeta_{s}\right]_{0, n-1} \\
& \left.A_{3}^{\prime}=\left[-j\left(c+\phi_{1} \eta_{G, g}\right) ; j \zeta_{1}, \cdots, j \zeta_{s}\right)\right]_{1, n} \\
& A_{4}^{\prime}=\left[-a-\eta_{G, g} \delta_{1}-(n-j)\left(c+\phi_{1} \eta_{G, g}\right) ; \epsilon_{1}+(n-j) \zeta_{1}, \cdots, \epsilon_{s}+(n-j) \zeta_{s}\right]_{1, k}
\end{aligned}
$$




$$
\begin{aligned}
& A_{5}^{\prime}=\left[1-a-b-\eta_{G, g}\left(\delta_{1}+\psi_{1}\right)-(2 n-j-1)\left(c+\phi_{1} \eta_{G, g}\right) ; \epsilon_{1}+\eta_{1}+(2 n-j-1) \zeta_{1}, \cdots\right. \\
& \left.\epsilon_{s}+\eta_{s}+(2 n-j-1) \zeta_{s}\right]_{1, k} \\
& B_{1}^{\prime}=\left(-c-\phi_{1} \eta_{G, g} ; \zeta_{1}, \cdots, \zeta_{s}\right), \cdots,\left(-c-\phi_{1} \eta_{G, g} ; \zeta_{1}, \cdots, \zeta_{s}\right) \\
& B_{2}^{\prime}=\left[1-a-\eta_{G, g} \delta_{1}-(n-j)\left(c+\phi_{1} \eta_{G, g}\right) ; \epsilon_{1}+(n-j) \zeta_{1}, \cdots, \epsilon_{s}+(n-j) \zeta_{s}\right]_{1, k} \\
& B_{3}^{\prime}=\left[-a-b-\eta_{G, g}\left(\delta_{1}+\psi_{1}\right)-(2 n-j-1)\left(c+\phi_{1} \eta_{G, g}\right) ; \epsilon_{1}+\eta_{1}+(2 n-j-1) \zeta_{1}, \cdots\right. \\
& \left.\epsilon_{s}+\eta_{s}+(2 n-j-1) \zeta_{s}\right]_{1, k}
\end{aligned}
$$

$$
\begin{aligned}
& B_{4}^{\prime}=\left[1-a-b-\eta_{G, g}\left(\delta_{1}+\psi_{1}\right)-(n+j-1)\left(c+\phi_{1} \eta_{G, g}\right) ; \epsilon_{1}+\eta_{1}+(n+j-1) \zeta_{1}, \cdots\right. \\
& \left.\epsilon_{s}+\eta_{s}+(n+j-1) \zeta_{s}\right]_{0, n-1}
\end{aligned}
$$

\section{Provided}

$$
\begin{aligned}
& \min \left\{\delta_{1}, \psi_{1}, \phi_{1}, \epsilon_{l}, \eta_{l}, \zeta_{l}\right\}>0 ; l=1, \cdots, s ; a, b, c \in \mathbb{C} \\
& A^{\prime}=\operatorname{Re}\left(a+\delta_{1} \eta_{G, g}\right)+\sum_{i=1}^{s} \epsilon_{i} \min _{1 \leqslant j \leqslant m_{i}} \operatorname{Re}\left(\frac{d_{j}^{(i)}}{\delta_{j}^{(i)}}\right)>0 \\
& B^{\prime}=\operatorname{Re}\left(b+\psi_{1} \eta_{G, g}\right)+\sum_{i=1}^{s} \eta_{i} \min _{1 \leqslant j \leqslant m_{i}} \operatorname{Re}\left(\frac{d_{j}^{(i)}}{\delta_{j}^{(i)}}\right)>0 \\
& C^{\prime}=\operatorname{Re}\left(c+\phi_{1} \eta_{G, g}\right)+\sum_{i=1}^{s} \zeta_{i} \min _{1 \leqslant j \leqslant m_{i}} \operatorname{Re}\left(\frac{d_{j}^{(i)}}{\delta_{j}^{(i)}}\right)>\operatorname{Max}\left\{-\frac{1}{n},-\frac{A^{\prime}}{n-1},-\frac{B^{\prime}}{n-1}\right\} \text { and } k \leqslant n . \\
& \left|\arg \left(z_{1} X_{\delta_{1}, \psi_{1}, \phi_{1}}\left(x_{1}, \cdots, x_{n}\right)\right)\right|<\frac{1}{2} \Omega_{1} \pi, \text { where } \Omega_{1}=\sum_{k=1}^{n^{(1)}} \alpha_{k}^{(1)}-\sum_{k=n^{(1)}+1}^{p^{(1)}} \alpha_{k}^{(1)}+\sum_{k=1}^{m^{(1)}} \beta_{k}^{(1)}-\sum_{k=m^{(1)}+1}^{q^{(1)}} \beta_{k}^{(1)}
\end{aligned}
$$

$\left|\arg \left(z_{i}^{\prime} X_{\epsilon_{i}, \eta_{i}, \zeta_{i}}\left(x_{1}, \cdots, x_{n}\right)\right)\right|<\frac{1}{2} U_{i} \pi$, where $U_{i}$ is defined by $(1.15)$ for $i=1, \cdots, s$

the series on the left-hand side of (4.1) converges absolutely.

Consider the above corollary, now the modified multivariable H-function reduces to H-function of one variable defined by Fox [3], we have.

Corollary 2.

$$
\int_{0}^{1} \cdots \int_{0}^{1} \prod_{i=1}^{k} x_{i} \prod_{i=1}^{n} x_{i}^{a-1}\left(1-x_{i}\right)^{b-1} \prod_{1 \leqslant j<k \leqslant n}\left|x_{j}-x_{k}\right|^{2 c} H\left(\mathrm{z}_{1} X_{\delta_{1}, \psi_{1}, \phi_{1}}\left(x_{1}, \cdots, x_{n}\right)\right)
$$




$$
\begin{gathered}
H_{p_{1}, q_{1}}^{m_{1}, n_{1}}\left(\mathrm{z}_{1}^{\prime} X_{\epsilon_{1}, \eta_{1}, \zeta_{1}}\left(x_{1}, \cdots, x_{n}\right)\right) \mathrm{d} x_{1} \cdots \mathrm{d} x_{n}=\sum_{G=1}^{m^{(1)}} \sum_{g=0}^{\infty} \phi_{1} \frac{z_{1}^{\eta_{G, g}}(-)^{g}}{\delta_{G} g !} \\
H_{p_{1}+3 n+2 k, q_{1}+2 n+2 k}^{m_{1}, n_{1}+3 n+2 k}\left(\begin{array}{c}
\mathrm{z}_{1}^{\prime} \\
\\
\mathrm{A}_{1}^{\prime \prime}, A_{2}^{\prime \prime}, A_{3}^{\prime \prime}, A_{4}^{\prime \prime}, A_{5}^{\prime \prime},\left(c_{j}^{(1)}, \gamma_{j}^{(1)}\right)_{1, p_{1}} \\
\cdot \\
\cdot \\
\\
\left.\mathrm{d}_{j}^{(1)}\right)_{1, q_{1}}, B_{1}^{\prime \prime}, B_{2}^{\prime \prime}, B_{3}^{\prime \prime}, B_{4}^{\prime \prime}
\end{array}\right)
\end{gathered}
$$

where

$$
\begin{aligned}
& A_{1}^{\prime \prime}=\left[1-a-\eta_{G, g} \delta_{1}-j\left(c+\phi_{1} \eta_{G, g}\right) ; \epsilon_{1}+j \zeta_{1}\right]_{0, n-1} \\
& A_{2}^{\prime \prime}=\left[1-b-\eta_{G, g} \psi_{1}-j\left(c+\phi_{1} \eta_{G, g}\right) ; \eta_{1}+j \zeta_{1}\right]_{0, n-1} \\
& \left.A_{3}^{\prime \prime}=\left[-j\left(c+\phi_{1} \eta_{G, g}\right) ; j \zeta_{1}\right)\right]_{1, n} \\
& A_{4}^{\prime \prime}=\left[-a-\eta_{G, g} \delta_{1}-(n-j)\left(c+\phi_{1} \eta_{G, g}\right) ; \epsilon_{1}+(n-j) \zeta_{1}\right]_{1, k} \\
& A_{5}^{\prime \prime}=\left[1-a-b-\eta_{G, g}\left(\delta_{1}+\psi_{1}\right)-(2 n-j-1)\left(c+\phi_{1} \eta_{G, g}\right) ; \epsilon_{1}+\eta_{1}+(2 n-j-1) \zeta_{1}\right]_{1, k} \\
& B_{1}^{\prime \prime}=\left(-c-\phi_{1} \eta_{G, g} ; \zeta_{1}\right), \cdots,\left(-c-\phi_{1} \eta_{G, g} ; \zeta_{1}\right) \\
& B_{2}^{\prime \prime}=\left[1-a-\eta_{G, g} \delta_{1}-(n-j)\left(c+\phi_{1} \eta_{G, g}\right) ; \epsilon_{1}+(n-j) \zeta_{1}\right]_{1, k} \\
& B_{3}^{\prime \prime}=\left[-a-b-\eta_{G, g}\left(\delta_{1}+\psi_{1}\right)-(2 n-j-1)\left(c+\phi_{1} \eta_{G, g}\right) ; \epsilon_{1}+\eta_{1}+(2 n-j-1) \zeta_{1}\right]_{1, k} \\
& B_{4}^{\prime \prime}=\left[1-a-b-\eta_{G, g}\left(\delta_{1}+\psi_{1}\right)-(n+j-1)\left(c+\phi_{1} \eta_{G, g}\right) ; \epsilon_{1}+\eta_{1}+(n+j-1) \zeta_{1}\right]_{0, n-1}
\end{aligned}
$$

\section{Provided}

$$
\begin{aligned}
& \min \left\{\delta_{1}, \psi_{1}, \phi_{1}, \epsilon_{1}, \eta_{1}, \zeta_{1}\right\}>0 ; a, b, c \in \mathbb{C} \\
& A^{\prime \prime}=\operatorname{Re}\left(a+\delta_{1} \eta_{G, g}\right)+\epsilon_{1} \min _{1 \leqslant j \leqslant m_{1}} \operatorname{Re}\left(\frac{d_{j}^{(1)}}{\delta_{j}^{(1)}}\right)>0 \\
& B^{\prime \prime}=\operatorname{Re}\left(b+\psi_{1} \eta_{G, g}\right)+\eta_{1} \min _{1 \leqslant j \leqslant m_{1}} \operatorname{Re}\left(\frac{d_{j}^{(1)}}{\delta_{j}^{(1)}}\right)>0 \\
& C^{\prime \prime}=\operatorname{Re}\left(c+\phi_{1} \eta_{G, g}\right)+\zeta_{1} \min _{1 \leqslant j \leqslant m_{1}} \operatorname{Re}\left(\frac{d_{j}^{(1)}}{\delta_{j}^{(1)}}\right)>\operatorname{Max}\left\{-\frac{1}{n},-\frac{A^{\prime \prime}}{n-1},-\frac{B^{\prime \prime}}{n-1}\right\} \text { and } k \leqslant n .
\end{aligned}
$$




$$
\begin{gathered}
\left|\arg \left(z_{1} X_{\delta_{1}, \psi_{1}, \phi_{1}}\left(x_{1}, \cdots, x_{n}\right)\right)\right|<\frac{1}{2} \Omega_{1} \pi, \text { where } \Omega_{1}=\sum_{k=1}^{n^{(1)}} \alpha_{k}^{(1)}-\sum_{k=n^{(1)}+1}^{p^{(1)}} \alpha_{k}^{(1)}+\sum_{k=1}^{m^{(1)}} \beta_{k}^{(1)}-\sum_{k=m^{(1)}+1}^{q^{(1)}} \beta_{k}^{(1)} \\
\left|\arg \left(z_{1}^{\prime} X_{\epsilon_{1}, \eta_{1}, \zeta_{1}}\left(x_{1}, \cdots, x_{n}\right)\right)\right|<\frac{1}{2} U_{1} \pi, \text { where } U_{1}=\sum_{j=1}^{m_{1}} \delta_{j}^{(1)}-\sum_{j=1+m_{1}}^{q_{1}} \delta_{j}^{(1)}+\sum_{j=1}^{n_{1}} \gamma_{j}^{(1)}-\sum_{j=n_{1}+1}^{p_{1}} \gamma_{j}^{(1)}
\end{gathered}
$$

the series on the left-hand side of (4.11) converges absolutely.

\section{Conclusion}

In this paper, we have evaluated a general Selberg integral involving the product of an expansion of multivariable I function defined by Prasad [5], modified multivariable H-function defined by Prasad and Singh [6] and class of multivariable polynomials defined by Srivastava [9] with general arguments. The formulae evaluated in this paper are very general nature. Thus, the results established in this research work would serve as a formula from which, upon specializing the parameters, as many as desired results involving the special functions of one and several variables can be obtained.

\section{References Références Referencias}

1. G. G Andrew and R. Askey, Special function. Cambridge. University. Press 1999.

2. P. J. Forrester and S. O. Warnaar. The importance of the Selberg integral, Bull. Amer. Math. Soc. (N.S.), 45(4) (2008), 489-534.

3. C. Fox, The G and H-functions as symmetrical Fourier Kernels, Trans. Amer. Math. Soc. 98 (1961), 395-429.

4. Y. N. Prasad, Multivariable I-function, Vijnana Parishad Anusandhan Patrika 29 (1986), 231-237.

5. Y. N. Prasad and A. K. Singh, Basic properties of the transform involving and Hfunction of r-variables as kernel, Indian Acad Math, (2) (1982), 109-115.

6. A. Selberg. Remarks on a multiple integral. Norsk Mat. Tidsskr, 26 (1944), 71-78.

7. H.M. Srivastava, A multilinear generating function for the Konhauser set of biorthogonal polynomials suggested by Laguerre polynomial, Pacific. J. Math. 177(1985), 183-191.

8. H. M. Srivastava and R. Panda, Some expansion theorems and generating relations for the H-function of several complex variables. Comment. Math. Univ. St. Paul. 24 (1975), 119-137.

9. H. M. Srivastava and R. Panda, Some expansion theorems and generating relations for the H-function of several complex variables II. Comment. Math. Univ. St. Paul. 25 (1976), 167-197.

10. H.M. Srivastava and N.P. Singh, The integration of certain products of the multivariable H-function with a general class of polynomials. Rend. Circ. Mat. Palermo. Vol 32 (No 2) (1983), 157-187. 